\title{
VII. Resümee
}

Im Chaos der ersten Nachkriegsjahre genossen die Kirchen, die als einzige gesellschaftliche Großverbände die NS-Diktatur und deren Zusammenbruch überstanden hatten, außerordentlich hohes Ansehen und übten als moralische Instanz auf weite Bevölkerungskreise eine allgemeine Orientierungsfunktion aus. Ihrer Haltung zur politischen Säuberung mußte deshalb eine besondere Bedeutung zukommen, war doch die Problematik der Entnazifizierung auf das engste verknüpft mit Fragen der politischen Moral, der (historischen) Gerechtigkeit und den Vorstellungen von Schuld und Sühne.

Bereits während der ersten Begegnungen und Unterredungen mit amerikanischen Besatzungsoffizieren brachten die deutschen Kirchenführer beider Konfessionen ihre Vorbehalte gegen die amerikanischen Neuordnungsvorstellungen zum Ausdruck. Vor allem die Ankündigung scharfer Entnazifizierungsmaßnahmen löste bei ihnen große Besorgnis aus. Denn aus ihrer politisch konservativen Orientierung heraus mußte jede umfassende Säuberung des Staats- und Verwaltungsapparats von NSDAP-Mitgliedern, unter denen sich viele kirchentreue Gemeindeglieder befanden, die liberal-bürgerlichen, sozialdemokratischen und kommunistischen Kräfte stärken und mithin zu einem wachsenden Einfluß jener Gesellschaftskonzeptionen führen, die eine Trennung von Staat und Kirche befürworteten. Vor besonderen Problemen standen die evangelischen Bischöfe, die 1933 die Machtergreifung Hitlers begeistert begrüßt hatten und sich nun schwer taten, wenn sie von der Militärregierung um Personalvorschläge zur Neubesetzung der Verwaltung gebeten wurden, denn sie konnten nur in geringem Umfang auf bewährte Persönlichkeiten aus dem demokratischen Spektrum der Weimarer Parteienlandschaft zurückgreifen.

Die Niederschriften über die ersten Gespräche mit amerikanischen Besatzungsoffizieren lassen die Vorbehalte prominenter evangelischer Kirchenführer aus den Reihen der Bekennenden Kirche gegenüber einer weltanschaulich pluralistischen und demokratisch verfaßten Gesellschaft deutlich erkennen. Ihr Hauptinteresse galt der Vertretung und Durchsetzung unmittelbar kirchlicher Anliegen. Sofern sie jedoch die Gelegenheit zu politischer Stellungnahme benutzten, dann um die Entnazifizierungspolitik zu kritisieren oder vor linken Kräften und Strömungen zu warnen. Konkrete Neuordnungskonzeptionen, die über den allgemeinen Wunsch hinausgingen, der Kirche wieder einen herausragenden gesellschaftlichen Einfluß zu sichern, lassen sich nicht feststellen. Die Unterredungen, vor allem mit Landesbischof Wurm und Niemöller, verstärkten die in der amerikanischen Militärregierung bereits bestehende Skepsis hinsichtlich der Demokratiebereitschaft des deutschen Protestantismus.

Das dringendste Problem, das sich den Kirchenführern unmittelbar nach dem Zusammenbruch des NS-Regimes stellte, war die Neuordnung der evangelischen Kirche, die sich während des Kirchenkampfes 1933/34 in die Bekennende Kirche und die Deutschen Christen gespalten hatte. Das volkskirchliche Potential war allerdings in den späteren Jahren in den meisten Landeskirchen von den ,,neutralen" Mittelgruppen gestellt worden. Da unter dem Druck des NS-Regimes und des Zweiten Weltkrieges die innerkirchlichen Gegensätze nicht hatten beigelegt werden können, brachen unmittelbar bei Kriegsende alle offenen Fragen und Kontroversen erneut auf. Erst das Einlenken der beiden Flügelgruppen der Bekennenden Kirche - der konfessionsbewußten Lutheraner um die 
Landesbischöfe Meiser und Marahrens einerseits und der von Niemöller angeführten Bruderräte andererseits - ermöglichte die von harten Auseinandersetzungen begleitete Kirchenkonferenz von Treysa Ende August 1945. Sie war maßgeblich das Werk des württembergischen Landesbischofs Wurm, dessen Integrationskonzept auf dem ,,Kirchlichen Einigungswerk" basierte. Wurm wurde nicht nur von der Okumene nachhaltig unterstützt, sondern auch indirekt von der amerikanischen Militärregierung favorisiert. Das bedeutendste Ergebnis der Kirchenkonferenz war die Gründung der Evangelischen Kirche in Deutschland (EKD), deren Rat ausschließlich Vertreter aus beiden Flügeln der Bekennenden Kirche angehörten.

Als der Rat der EKD mit der primär für die Okumene bestimmten Stuttgarter Schulderklärung vom 18./19. Oktober 1945 erstmals öffentlich in Erscheinung trat, löste er in den Kirchengemeinden einen Sturm der Entrüstung und Empörung aus. Auf die allseits konstatierte Vertrauenskrise reagierte der Rat mit wachsender Kritik an der alliierten Besatzungspolitik, die die Solidarität der Kirche mit dem deutschen Volk kundtun sollte und zu einer problematischen Anpassung an die Stimmung im Kirchenvolk führte. Der insbesondere von Karl Barth geforderten Erkenntnis der strukturellen Fehlentwicklung der deutschen Gesellschaft standen deutschnationale Denkweisen und eine dämonologisierende Interpretation des Nationalsozialismus entgegen, die sich in vielfältiger Weise mit der traditionellen theologischen Weltdeutung der Moderne als eines fortschreitenden Verfallsprozesses verbanden. Die von kleinen bruderrätlichen Zirkeln vorangetriebene Konkretisierung des Schuldbekenntnisses, wie sie sich im August 1947 im ,Darmstädter Wort" niederschlug, wurde aber auch von den Bruderräten weitgehend zurückgewiesen.

Das selbstbewußte Auftreten der Kirchenführer gegenüber den Besatzungsmächten gründete nicht zuletzt in der Uberzeugung, daß die Kirche aus eigener Kraft eine Zeit schwerer Bedrängnis überstanden und trotz manchen Versagens und Fehlens erfolgreich dem Totalitätsanspruch der NS-Diktatur widerstanden habe. Die Erkenntnis der eigenen Anfälligkeit für den Nationalsozialismus trat demgegenüber zurück, partielle Selbstbehauptung wurde als prinzipielle Absage an das NS-Regime ausgegeben. Der kritischen Auseinandersetzung mit der NS-Vergangenheit und der ,Schuld der Kirche" waren deshalb in den ersten Nachkriegsjahren enge Grenzen gesetzt.

Als im Sommer 1945 die Konturen der amerikanischen Säuberungspolitik sichtbar wurden, reagierten die Kirchenführer mit scharfen Protesten gegen die schematische Entlassungspraxis, die der Lebenswirklichkeit im Dritten Reich nicht gerecht werden konnte und von zahlreichen Ungerechtigkeiten begleitet war. Die Kritik richtete sich nicht gegen die Entlassung eines kleinen Kreises hoher NS-Funktionäre, sondern dagegen, daß die amerikanische Militärregierung auf der pauschalen Entlassung aller bestand, die vor dem 1. Mai 1937 der NSDAP beigetreten waren, und damit dem für die kirchliche Beurteilung ausschlaggebenden Kriterium der Kirchentreue keine Rechnung trug. Der befürchtete Verlust konservativer Machtpositionen im öffentlichen Dienst und die wiedererwachte Furcht vor der politischen Linken bewirkten eine partielle Uberwindung althergebrachten konfessionellen Mißtrauens und führten bereits am 20. Juli 1945 zu einem gemeinsamen Protest Kardinal Faulhabers und Landesbischof Meisers, der den Auftakt für eine lange Reihe von Eingaben und Petitionen zugunsten ehemaliger Nationalsozialisten bildete.

Zweifellos hatten die Kirchenführer der Militärregierung die politische Einsicht voraus, daß viele NSDAP-Mitglieder keine überzeugten Nationalsozialisten mehr waren 
und im Interesse des sozialen Friedens früher oder später wieder integriert werden mußten. Dieses Anliegen vermochten sie, wenn sie - wie alle politischen Gruppierungen - für eine differenziertere Beurteilung eintraten, jedoch nur unzureichend zu vermitteln. Der frontale Aufgriff auf die politische Säuberung und die oft recht apologetischen Rechtfertigungsversuche mußten die Glaubwürdigkeit des kirchlichen Engagements schwer erschüttern; zumal die Kritik der evangelischen Kirchenführer schon im Mai 1945 mit den ersten, noch sehr begrenzten Säuberungsmaßnahmen der Militärregierung eingesetzt hatte.

Eine neue Phase der Entnazifizierung leitete in der amerikanischen Besatzungszone das im März 1946 erlassene Gesetz zur Befreiung von Nationalsozialismus und Militarismus ein. Es führte die von den Deutschen geforderte Einzelfallprüfung nach Maßgabe der individuellen Verantwortung und der tatsächlichen Gesamthaltung ein und stellte insofern einen bedeutsamen Fortschritt dar. Als politische Säuberung konnte die Entnazifizierung unter deutscher Verantwortung jedoch nur Erfolg haben, solange sie auf einen - durchaus weit definierten - Kreis von Schlüsselstellungen beschränkt blieb und nicht zur Massenanklage ausuferte. Aus der nunmehrigen Uberprüfung aller Mitglieder von NS-Organisationen und der Verlagerung politischer Säuberungsvorgaben auf die entpolitisierte Ebene des gerichtsähnlichen Spruchkammerverfahrens resultierte unweigerlich der sich über Jahre hinschleppende, viel zu langwierige Prozeß einer bürokratischen Massenentnazifizierung, die de facto zu einer Massenrehabilitierung wurde.

Im Mittelpunkt der Kritik der evangelischen Kirche stand nicht die verfehlte Anlage des Befreiungsgesetzes, sondern die unvermindert beibehaltene Ablehnung der politischen Säuberung an sich als grundsätzlichem Unrecht. Bereits am 26. April 1946, als noch kein einziges Spruchkammerurteil vorlag, sprach Wurm als Ratsvorsitzender der EKD in einem Schreiben an die amerikanische Militärregierung und die deutschen Länderregierungen dem Gesetz jegliche politische und moralische Berechtigung mit der rechtspositivistischen Argumentation ab, daß die , Handlungen und Gesinnungen, die heute verurteilt werden, vom damaligen Gesetzgeber als rechtmäßig und gut eingeschätzt" worden seien. Die Kirche könne ,, nicht anerkennen, daß eine menschliche Obrigkeit nunmehr zu strafen unternimmt, was allein nach göttlichem Recht als Unrecht zu gelten" habe"

Diese frühzeitig vollzogene Ablehnung des Befreiungsgesetzes wurde nicht mehr grundsätzlich revidiert. Der Kritik, wie sie in offiziellen Stellungnahmen von Kirchenleitungen und öffentlichen Äußerungen von Kirchenführern zur Geltung kam, lag auch in späteren Jahren keine fundierte Anlayse des tatsächlichen Entnazifizierungsprozesses zugrunde. Sie enthielt daher kaum konstruktive Momente, sondern schürte eher Ressentiments und Emotionen, wo Differenzierung dringend notwendig gewesen wäre. Das hervorstechendste Merkmal war dabei die Verkennung des Rehabilitierungscharakters des deutschen Spruchkammerwesens. Mochte dies bei der Verabschiedung des Befreiungsgesetzes für die Offentlichkeit noch nicht deutlich erkennbar sein, so ließ spätestens die Spruchkammerpraxis an der großzügigen Rehabilitierung keinen Zweifel mehr, wie die Statistiken zum Leidwesen der Militärregierung eindeutig belegen. Der zunehmende Verlust an politischem Augenmaß und sachgerechter Kritik erreichte Anfang 1948 im Boykott-Aufruf Niemöllers, der eine heftige öffentliche Kontroverse auslöste, seinen sichtbarsten Ausdruck.

$1 \mathrm{Vgl} . \mathrm{S.} 71 \mathrm{ff}$. 
Die politische Verantwortung der Kirche, ihr ,, Wächteramt“, wurde in den ersten Nachkriegsjahren kaum theologisch reflektiert, sondern mehr nach Maßgabe politischer Grundorientierungen ausgeübt. Entgegen den Intentionen der Kirchenführer, die ihrem Volk in der Stunde der Not beistehen, keinesfalls aber nachträglich das NS-Regime rechtfertigen wollten, trugen ihre Stellungnahmen zur allgemeinen Verdrängung dieser Vergangenheit bei. Problematisch war nicht, daß die Kirche für das Millionenheer der Mitläufer eintrat, sondern ihre Argumentationsweise, die auf pauschale Rechtfertigung und Entlastung von jeglicher Mitverantwortung abzielte. Die Proteste der evangelischen Kirche spiegelten die weitverbreitete Ablehnung des Befreiungsgesetzes wider, dessen viel zu breite Anlage zur Solidarisierung der Mitläufer mit überzeugten NS-Aktivisten führen mußte, und verstärkten sie zugleich mit der moralischen Autorität der Kirche, da die Entnazifizierung nicht als eine Grundsatzfrage der politischen Moral und des demokratischen Neubeginns thematisiert wurde.

Die Ablehnung der Entnazifizierung wurde von sämtlichen kirchenpolitischen Gruppierungen des deutschen Protestantismus, mit Ausnahme der kleinen Kirchlich-theologischen Sozietät Württembergs, mitgetragen. Auch diejenigen bruderrätlichen Kreise, die 1947 im Darmstädter Wort ein Manifest zur gesellschaftspolitischen Neuorientierung vorgelegt hatten, sahen keinen Anlaß, sich von der offiziellen Haltung der evangelischen Kirche zu distanzieren. Differenzen ergaben sich aber hinsichtlich der Selbstreinigung der Kirche. Nicht zuletzt gilt es festzuhalten, daß der massiven Fürsprache für ehemalige NSDAP-Mitglieder kein Wort zugunsten einer Wiedergutmachung an den Opfern des NS-Regimes gegenüberstand.

Zwar liegen keine statistischen Angaben hinsichtlich der Konfessionszugehörigkeit der Entnazifizierten vor, doch wird man angesichts der wesentlich höheren Wahlerfolge der NSDAP in protestantischen Gebieten auf eine größere Betroffenheit des evangelischen Bevölkerungsteils schließen dürfen. Die traditionelle soziale Milieuverengung der evangelischen Kirche auf mittelständische und ländlich-bäuerliche Schichten sowie auf adelige Kreise, die sich besonders in der Zusammensetzung der Pfarrerschaft und der Synoden niederschlug, bewirkte eine hohe soziale Identität und Identifikation mit den von der Entnazifizierung bedrohten Schichten. Im Mittelpunkt der politischen Säuberung standen die Angestellten und Beamten des öffentlichen Dienstes, dem auch die Väter von einem Drittel der evangelischen Pfarrer angehörten, während nur 3,4 Prozent einem Arbeiter- oder unselbständigen Handwerkerhaushalt entstammten ${ }^{2}$. Von kaum zu unterschätzender Bedeutung ist weiterhin, daß die evangelische Kirche aufgrund des - im Vergleich zur katholischen Kirche - hohen Anteils belasteter Pfarrer auch als Institution unmittelbar betroffen war.

In den hessischen Teilkirchen (Hessen-Darmstadt, Nassau und Frankfurt) galten nach den Kriterien des Befreiungsgesetzes 226 von 645 aktiven Pfarrern als belastet, in der Bremischen Kirche waren es sogar 51 von 55 . Doch selbst in den ,,intakten" süddeutschen Landeskirchen, in denen die Deutschen Christen keinen Führungswechsel erzwingen konnten, hatten 302 von rund 1100 aktiven bayerischen Pfarrern der NSDAP oder anderen NS-Organisationen angehört. In Württemberg waren es 333 von 1197, einschließlich der französischen Besatzungszone. Die badische Landeskirche meldete für

2 Pfarrerstatistik nach den Berufen der Väter von 1950, in: KJ 1955, S. 400f. Vgl. auch Balzer, Kirche. 
den Bereich der US-Zone 143 belastete Pfarrer von 341 aktiven. Hinsichtlich der Parteimitgliedschaft ergibt sich folgendes Bild: In Bayern waren von insgesamt 429 belasteten Pfarrern mindestens 209 NSDAP-Mitglieder gewesen. In Hessen gab die Kirchenleitung 1948103 Parteimitglieder an, und von den 96 belasteten Pfarrern der württembergischen Landeskirche, die in der französischen Zone tätig waren, hatten 41 der NSDAP angehört. In Bremen hatten sich von 51 belasteten Pfarrern neun der NSDAP angeschlossen ${ }^{3}$. Sicherlich wird man die Mitgliedschaft in der NS-Volkswohlfahrt nicht mit dem Beitritt zur SA oder zur NSDAP gleichsetzen können. Auch war nicht jeder Parteigenosse ein überzeugter Nationalsozialist, wie umgekehrt nicht jeder NS-Aktivist eingeschriebenes Mitglied war. Und viele von denen, die sich 1933/34 für das NS-Regime begeistert hatten, änderten im Kirchenkampf ihre Überzeugung. Trotz aller angebrachten Vorbehalte wird man die Mitgliedszahlen dennoch als Beleg für die hohe Anfälligkeit der evangelischen Pfarrerschaft für den Nationalsozialismus werten müssen.

Sie lenken den Blick auf die psychologischen und gesellschaftlichen Hemmnisse, die unabhängig von den gravierenden Fehlern der amerikanischen Entnazifizierungspolitikauf deutscher Seite jeder umfassenden Personalsäuberung im Wege standen. Die Herrschaft des Nationalsozialismus hatte nicht ausschließlich auf Manipulation und Terror beruht, sondern auch auf einem hohen Maß sozialer Akzeptanz. ,,Volksgemeinschaft" war für viele Deutsche keine ideologische Leerformel gewesen, sondern „Aufruf zur Uberwindung der Relikte vorbürgerlicher, vorindustrieller sozialer Hierarchien und Normen, Aufruf zur Bildung einer modernen, mobilen bürgerlich-nationalen Massengesellschaft ${ }^{\star \star 4}$. Die NS-Propaganda, so dürftig ihre intellektuelle Qualität sein mochte, hatte sich - zusammen mit sozialen Modernisierungsschüben - als ein wirksames Instrument zur Erhaltung politischer Massenloyalität erwiesen. Zwar hatte die positive Integrationskraft des Nationalsozialismus nach Stalingrad erheblich nachgelassen, war die nationale Begeisterung einer mehr apathisch-resignativen Grundstimmung gewichen, doch blieb die Identifikation von Nation und NS-Regime bis fast zuletzt erhalten.

Begeisterung, Anpassung und partielle Resistenz waren in der NS-Zeit in vielfältiger Weise verschmolzen, so daß 1945 kaum jemand guten Gewissens einen moralischen Rigorismus verfechten konnte. Die eher dumpf empfundene eigene Verstrickung und Mitschuld, auch im Sinne unterlassener Handlungen, förderte abstrakte Schuldzuweisungen an die ,Hitler-Clique“ oder an namenlose Dämonen. Die deutschen NS-Gegner gehörten zwar 1945, sofern sie überlebt hatten oder bereits aus der Emigration zurückgekehrt waren, zu den innenpolitischen Siegern, doch fanden sie nur wenig Rückhalt in der Bevölkerung. In dieser Situation mußte jede tiefgreifende Säuberung den Charakter einer , ,künstlichen Revolution“5 annehmen, die sich primär aus der Dispositionsfreiheit der Siegermächte ableitete. Aber auch die Handlungsfreiheit der amerikanischen Militärregierung, einer liberalen Besatzungsdiktatur, fand ihre Grenzen am Gesellschaftssystem der Sieger wie an der trägen Beharrungskraft gewachsener Strukturen und Mentalitäten bei den Besiegten. Ohne die Zustimmung und Mitarbeit der Deutschen selbst ließ sich

${ }^{3}$ Vgl. Kap. IV/5 und Kap. V/1-3.

4 Broszat, Struktur, S. 66.

${ }^{5}$ So beispielsweise 1947 Knappstein, leitender Mitarbeiter im hessischen Befreiungsministerium, Die versäumte Revolution. Vgl. Henke, Grenzen. 
eine politische Säuberung wohl administrativ verordnen, nicht aber effektiv und dauerhaft wirksam durchführen.

Die Selbstreinigung in den evangelischen Landeskirchen kann als Testfall für den deutschen Säuberungswillen gelten: War doch allein den Kirchen von der Militärregierung das Privileg zugestanden worden, ihre internen Verhältnisse ohne Eingriffe von außen zu regeln. Hier besaßen die Kirchenführer volle Handlungsfreiheit, hier mußte sich der proklamierte Wille zum Neuanfang erweisen. Die untersuchten Landeskirchen der US-Zone repräsentieren die Spannbreite des deutschen Protestantismus wie auch den unterschiedlichen Verlauf des Kirchenkampfes und der Neuordnung nach 1945. Der bayerische Landesbischof Meiser zählte zu den dominierenden Persönlichkeiten des konservativ geprägten Luthertums und übernahm 1948 die Leitung der Vereinigten Evangelisch-Lutherischen Kirche Deutschlands. Der württembergische Bischof Wurm war zugleich Ratsvorsitzender der EKD. Niemöller schließlich vertrat den bruderrätlichen Flügel der Bekennenden Kirche in Hessen und Nassau. In scharfem Kontrast zur Kontinuität der ,,intakten" süddeutschen Landeskirchen zerfiel die deutsch-christlich regierte Landeskirche Nassau-Hessen unmittelbar nach dem Zusammenbruch des NS-Regimes in ihre drei ursprünglichen Teilgebiete (Hessen-Darmstadt, Nassau und Frankfurt). Mit zum Untersuchungsgebiet gehört ferner die unierte Bremische Evangelische Kirche, in der ebenfalls die Deutschen Christen die Macht übernommen hatten.

Wie die Untersuchung zeigen konnte, waren die süddeutschen Landesbischöfe Meiser und Wurm zu keinen einschneidenden Säuberungsmaßnahmen bereit und verteidigten mit Erfolg auch schwer belastete NSDAP-oder DC-Pfarrer gegen Anfechtungen der Militärregierung oder aus den eigenen Reihen. Einen ähnlichen Weg versuchte die evangelische Kirche in Bremen zu gehen, die jedoch als kleine Landeskirche einem Konflikt mit der Militärregierung nicht gewachsen war und sich Mitte 1946 dem Entlassungsdruck beugte. Das zentrale Verteidigungsargument gegen die von außen geforderte Säuberung war der Bezug auf die Autonomie der Kirche: nur der Kirche stehe das Recht zur Entscheidung darüber zu, wer als Pfarrer tätig sein dürfte. Die aus dem Kirchenkampf geläufige Argumentation verkannte, daß die Mitgliedschaft in der NSDAP oder in anderen NS-Organisationen eine politische Entscheidung darstellte, zu der der einzelne Pfarrer nicht in Ausübung seines geistlichen Amtes gezwungen war, sondern die er in seiner Eigenschaft als Staatsbürger zu verantworten hatte. Wie insbesondere die Fallstudie zur bayerischen Landeskirche zeigt, nahmen die Kirchenleitungen vielfach massiven Einfluß auf die Verfahren gegen belastete Pfarrer und intervenierten auf höchster politischer Ebene, um eine Revision unliebsamer Spruchkammerurteile zu erreichen.

Lediglich in den hessischen Teilkirchen betrieben die Bruderräte anfangs mit der Einsetzung eigener Untersuchungsausschüsse und scharfen Säuberungsrichtlinien, die den widerchristlichen Charakter des Nationalsozialismus hervorhoben, eine energische Selbstreinigung. Die zeitweilige Suspendierung von 88 Pfarrern beruhte allerdings nicht auf einem innerkirchlichen Konsens, sondern resultierte aus der handstreichartigen Übernahme der Kirchenleitungen durch die Bruderräte unmittelbar nach Kriegsende und dem Druck der Militärregierung. Der Verlauf der unterbliebenen bzw. wenig später zurückgenommenen Selbstreinigung ${ }^{6}$ entsprach dem allgemeinen Trend und verweist dar-

${ }^{6}$ Rehabilitiert wurden auch prominente DC-Führer: $z$. B. amtierte der braunschweigische DC-Bischof Wilhelm Beye, NSDAP-Mitglied seit 1922 und von 1941-45 NSDAP-Gauschulungsleiter 
auf, daß die NS-Gegner als gesellschaftlicher Träger eines autonomen Selbstreinigungsprozesses zu schwach waren.

Die Nachzeichnung einiger Spruchkammerfälle vermittelt schließlich einen Einblick in den konkreten Ablauf des Entnazifizierungsverfahrens und die Fragwürdigkeit zahlreicher Spruchkammerurteile. Die Fälle spiegeln die Bandbreite unterschiedlicher Belastungen wider und geben Aufschluß über die politischen und mentalen Dispositionen von Pfarrern, die die politische Betätigung für den Nationalsozialismus mit ihrem priesterlichen Auftrag für vereinbar gehalten hatten. Für die meisten dieser Pfarrer stellte der Kirchenkampf einen Loyalitätskonflikt dar, den sie ohne größeres Zögern zugunsten der Kirche entschieden. Eine prinzipielle Absage an das NS-Regime war damit jedoch nur in seltenen Fällen verbunden. Die Rechtfertigungen wie die Flut bereitwillig erteilter , Persilscheine" verdeutlichen, daß auch nach 1945 der Nationalsozialismus keineswegs mit Terror, Krieg und Verbrechen gleichgesetzt wurde. Das Geflecht kollegialer, sozialer und familiärer Bindungen und Rücksichtnahmen ließ den Säuberungswillen, soweit ihn einzelne Spruchkammern entwickelten, spätestens im Berufungsverfahren weitgehend ins Leere laufen.

Als Institution stärkte die evangelische Kirche auch nach 1945 das konservative Lager, da die erhoffte Rechristianisierung von Staat und Gesellschaft nur mit diesen Kräften angegangen werden konnte. In bemerkenswerter Ubereinstimmung lautet denn auch das Urteil der Literatur, die evangelische Kirche habe zur Förderung konservativ-restaurativer Bestrebungen beigetragen und emanzipatorische Neuansätze verhindert ${ }^{7}$. Daneben darf aber nicht übersehen werden, daß die im Dritten Reich leidvoll gewonnene Erkenntnis der Priorität rechtsstaatlicher Normen ein neues Verständnis der Aufgaben von Staat und Kirche bewirkte und einer blinden Staats- und Machtverehrung nunmehr wirksame Grenzen setzte. Die innere Distanz des Protestantismus zum gesellschaftlichen Pluralismus der Bundesrepublik wurde aber erst in den fünfziger und sechziger Jahren im Zuge einer Neubestimmung des eigenen Standorts abgebaut. Auch die Hinwendung zu einem sozial aufgeklärten Konservatismus ist nicht zu übersehen und kann nicht ausschließlich auf das Motiv der Abwendung des Kommunismus reduziert werden. Verweisen die vielfach steckengebliebenen Reformansätze in den ersten Nachkriegsjahren auf das anhal-

in Berlin, ab 1950 wieder als Pfarrer in Schleswig-Holstein. Der thüringische DC-Bischof Hugo Rönck, NSDAP-Mitglied seit 1925, amtierte ab 1947 als Pfarrer in Eutin. Siegfried Leffler, der Begründer der DC-Nationalkirchlichen Bewegung und Leiter des Eisenacher DC- ,Instituts zur Erforschung des jüdischen Einflusses auf das deutsche kirchliche Leben", wurde 1949 als Amtsaushilfe in Bayern bestätigt. Theologieprofessor Walter Grundmann, der wissenschaftliche Leiter des Instituts, wurde nach 1945 Rektor des Katechetischen Seminars in Eisenach. Dr. Heinz Hunger, der Geschäftsführer des Instituts, übernahm die Schriftleitung der Fachzeitschrift ,,Der evangelische Religionslehrer an Berufsschulen". Joachim Hossenfelder, Gründer und erster Reichsleiter der DC, verwaltete bereits 1946 wieder eine Pfarrstelle im brandenburgischen Vehlow. DC-Führer Reinhold Krause, dessen Rede den Sportpalastskandal ausgelöst hatte, war 1951 in Konstanz wieder im Schuldienst.

7 Vgl. Conway, Rolle, S. 369 f.; Fischer, Kirche, S. 122 ff., 191 ff.; Hein-Janke, Protestantismus, S. 349 ff.; Greschat, Kirche, S. 106; Greschat, Kontinuität, S. 94; Noormann, Protestantismus, Bd. 1, S. 276 ff.; Jochmann, Kirche, S. 652; Scheerer, Kirche, S. 230 ff., 277 f.; mit polemischer Zuspitzung Prolingheuer, Kirchengeschichte, S. $117 \mathrm{ff}$. Vgl. dazu ausführlicher Vollnhals, Kirche zwischen Traditionswahrung. 
tende Gewicht nationalprotestantischer Traditionen und Hypotheken, so zeigt andererseits allein die Existenz des Stuttgarter Schuldbekenntnisses und des Darmstädter Wortes, daß sich die Gesamtentwicklung der evangelischen Kirche nicht einfach mit dem strapazierten Begriff der Restauration fassen läßt. Wie in anderen gesellschaftlichen Bereichen erweist sich auch am Beispiel der evangelischen Kirche, daß die politische Zäsur des Jahres 1945 wesentlich elementarer war als die mentalen, sozialen und gesellschaftlichen Einschnitte der unmittelbaren Nachkriegsjahre. 